\title{
High-Tech-Metalle in ostalpinen Buntmetallsulfidvorkommen: geologische Faktoren ihrer Anreicherung, Verteilung und Mobilisation (drei MRI-Projekte 2015-2019)
}

\author{
Thomas Angerer ${ }^{1}$, Frank Melcher ${ }^{2}$, Peter Onuk ${ }^{2,3}$, Peter Tropper ${ }^{1}$, Albin Volgger ${ }^{1,4}$ und Patrick Gasteiger ${ }^{1}$ \\ ${ }^{1}$ Mineralogisches-Petrographisches Institut, Universität Innsbruck, Innsbruck, Österreich \\ ${ }^{2}$ Department Angewandte Geowissenschaften und Geophysik, Montanuniversität Leoben, Leoben, Österreich \\ ${ }^{3}$ NAWI Geozentrum Graz, Bereich Geochemie und Petrologie, Graz, Österreich \\ ${ }^{4}$ Bergbau Museum Ridnaun, Ratschings, Italien
}

Eingegangen 15. Februar 2021; angenommen 24. Februar 2021; online publiziert 18. März 2021

\begin{abstract}
Zusammenfassung: Die Ergebnisse dreier MRI Projekte 2015-2019 (Leoben und Innsbruck) werden hier zusammenfassend dargestellt. Der Fokus liegt auf den Ergebnissen des Innsbrucker Projekts, in dem sedimentär-exhalative Erze des Ötztal-Stubai-Kristallins (ÖSK) als beispielhaftes Mineralsystem erforscht wurden, um den komplexen Einfluss geologischer Prozesse auf die Anreicherung bzw. mineralogische Verteilung von Spurenelementen zu verdeutlichen.
\end{abstract}

Die umfangreichen Datensätze aus vielen ostalpinen Bergbaurevieren erlauben Aussagen über die Präferenz von Spurenelementen in verschiedenen Typen von $\mathrm{Fe}-\mathrm{Cu}-\mathrm{Zn}$ Sulfidparagenesen. Die Elemente Mn, Ga, Se und Cd werden präferentiell in Sphalerit eingebaut; $\mathrm{Co}, \mathrm{Ni}$ in Pyrit und Pyrrhotin; As in Pyrit; Ag und Sn im Chalkopyrit. In karbonatgebundenen $\mathrm{Pb}-\mathrm{Zn}$ Erzen wird $\mathrm{Ge}$ in Sphalerit und in Cu-reichen Erze auch in Chalkopyrit eingebaut. Indium ist gleichfalls entweder an Sphalerit oder an Chalkopyrit gebunden. Mit der Temperaturabhängigkeit der Sphaleritzusammensetzung gehen auch systematische Trends in der Zusammensetzung von Begleitsulfiden (v.a. Chalkopyrit) einher. In den Erzen des ÖSK wurden komplexe Verteilungsabhängigkeiten seltener Metalle von syngenetischen Mineralisations- sowie metamorph-metasomatischen Remobilisierungs-Prozessen nachgewiesen. Die Faktoren lithostratographischer Kontext, Proximalität zum (nicht aufgeschlossenen) Exhalationszentrum, und metasomatische Remobilisation und Partitionierung sind wichtige Fak-

\footnotetext{
T. Angerer $(\bowtie)$

Mineralogisches-Petrographisches Institut,

Universität Innsbruck,

6020 Innsbruck, Österreich

Thomas.angerer@uibk.ac.at
}

toren für die Höffigkeit eines Vorkommens. Metamorphe Überprägung zeigt Auswirkungen auf die mineralogische Metall-Partitionierung, jedoch ohne nennenswerten Effekt auf die Höffigkeit der High-Tech-Metalle.

Schlüsselwörter: Kritische Rohstoffe, Indium, Germanium, Gallium, Sphalerit, Chalkopyrit, Ötztal-Stubai-Kristallin

High-Tech Metals in Eastern Alpine Base Metal Sulfide Ores: Geological Factors of Their Enrichment, Distribution, and Mobilization (Three MRI Projects 2015-2019)

Abstract: The results of three MRI projects 2015-2019 (Leoben and Innsbruck) are summarized here. The focus is on the results of the Innsbruck project, in which sedimentary-exhalative ores of the Ötztal-Stubai crystalline block (ÖSK) were investigated, as an exemplary mineral system to illustrate the complex influence of geological processes on the enrichment and mineralogical distribution of trace elements.

The extensive data sets from numerous Eastern Alpine mining areas allow statements to be made about the preference of trace elements in various types of $\mathrm{Fe}-\mathrm{Cu}-\mathrm{Zn}$ sulfide parageneses. The elements $\mathrm{Mn}, \mathrm{Ga}, \mathrm{Se}$, and $\mathrm{Cd}$ are preferentially incorporated into sphalerite; $\mathrm{Co}, \mathrm{Ni}$ in pyrite and pyrrhotite; As in pyrite; $\mathrm{Ag}$ and $\mathrm{Sn}$ in chalcopyrite. In carbonate-bound $\mathrm{Pb}-\mathrm{Zn}$ ores, $\mathrm{Ge}$ is incorporated in sphalerite and in $\mathrm{Cu}$-rich ores also in chalcopyrite. Indium is also hosted in either sphalerite or chalcopyrite. The temperature dependence of the sphalerite composition is accompanied by systematic trends in the composition of cogenetic sulfides (especially chalcopyrite). In the ores of the ÖSK, complex distribution dependencies of rare metals from syn- 


\begin{tabular}{|c|c|c|c|c|}
\hline \multicolumn{5}{|c|}{$\begin{array}{l}\text { TABELLE } 1 \\
\text { Übersicht der drei MRI-Projekte }\end{array}$} \\
\hline Leitung & Projekt & Zeitraum & Fokus & Datensätze \\
\hline \multirow[t]{2}{*}{$\begin{array}{l}\mathrm{MU} \\
\text { Leoben }\end{array}$} & $\begin{array}{l}\text { "Identifikation und Klassifizierung } \\
\text { potenzieller Hochtechnologie-Me- } \\
\text { tall Ressourcen in ostalpinen Blei- } \\
\text { Zinklagerstätten" }\end{array}$ & $2015-2017$ & $\begin{array}{l}\text { Einbezug einer Vielzahl von Vor- } \\
\text { kommen in einen umfassenden } \\
\text { Ansatz }\end{array}$ & \multirow[t]{2}{*}{$\begin{array}{l}450 \text { Proben, }>50 \\
\text { Vorkommen in den Ost- } \\
\text { und Südalpen }\end{array}$} \\
\hline & $\begin{array}{l}\text { "Laser Ablation-ICP-MS Bestimmung } \\
\text { von Spurenelementen in Eisen- und } \\
\text { Kupfersulfiden österreichischer La- } \\
\text { gerstätten" }\end{array}$ & 2017-2019 & $\begin{array}{l}\text { Erweiterung des regionalen } \\
\text { Datensatzes um co-genetische } \\
\text { Chalkopyrite, Pyrite und Pyrrho- } \\
\text { tite }\end{array}$ & \\
\hline $\begin{array}{l}\text { Uni Inns- } \\
\text { bruck }\end{array}$ & $\begin{array}{l}\text { "Das Pb-Zn-Cu-Fe-Metallsystem im } \\
\text { Ötztal-Stubai-Kristallin: Chemische } \\
\text { Untersuchungen zu Herkunft und } \\
\text { Anreicherung von HT-Metallen mit } \\
\text { einer Bewertung des ökonomischen } \\
\text { Potentials" }\end{array}$ & 2016-2019 & $\begin{array}{l}\text { Vorkommen im Ötztal-Stubai- } \\
\text { Kristallin (ÖSK), Zusammenhang } \\
\text { von Anreicherung bzw. Parti- } \\
\text { tionierung von HT-Metallen in } \\
\text { Sulfiden und der Erzgenese und } \\
\text {-Metamorphose }\end{array}$ & $\begin{array}{l}62 \text { Proben aus } 18 \text { Vorkom- } \\
\text { men. } \\
+\sim 50 \text { Proben aus Schnee- } \\
\text { berg (MSc Albin Volgger } \\
\& \text { Patrick Gasteiger 2018) } \\
\text { (siehe Fußnote 1) }\end{array}$ \\
\hline
\end{tabular}

genetic mineralization and metamorphic-metasomatic remobilization processes have been demonstrated. The factors of lithostratigraphic context, proximity to the (not outcropping) exhalation center, and metasomatic remobilization, and partitioning are important factors for the high-tech metal abundance in a given ore occurrence. Metamorphic overprinting shows effects on the mineralogical metal partitioning but without any notable effect on the abundances of high-tech metal.

Keywords: Critical raw materials, Indium, Germanium, Gallium, Sphalerite, Chalcopyrite, Ötztal crystalline basements

\section{Einleitung}

\subsection{Problemstellung und Ausgangslage}

Auf Empfehlung der "Ad-Hoc Working Group on defining critical raw materials" der Europäischen Kommission wird eine Reihe von seltenen Metallen als Rohstoffe eingestuft, deren Versorgung für Länder der EU in Zukunft als kritisch gilt. $\mathrm{Zu}$ diesen Metallen gehören unter anderen die sogenannten High Tech-Metalle (HT-Metalle) Indium (In), Germanium (Ge), und Gallium (Ga), im weiteren Sinne auch Cobalt (Co) und Antimon (Sb), die z. B. in Solarpanel-Halbleitern, Licht \& Optik (LCD, LED) und Speziallegierungen Anwendung finden und deren natürliches mineralisches Vorkommen in ökonomischen Konzentrationen auf wenige Lagerstättentypen beschränkt ist. Hinsichtlich des Potentials für diese Metalle wird im Rohstoffplan Österreichs den Kupfer-Blei-Zink Lagerstätten der Ostalpen ein sicherungswürdiger bzw. bedingt sicherungswürdiger Status zugeschrieben [1]. Obwohl die recht umfassenden petrographischen und bereichsweisen geochemischen Analysen der verschiedenen Buntmetallvorkommen zu einem akzeptablem Wissen bezüglich der Metallogenese geführt haben [2], sind die Spurenelementverteilungen und deren Anreicherungsprozesse weitestgehend unerforscht $[3,4]$.

Im Zuge der GBA Mineralrohstoffinitiative wurden von 2015-2019 drei Forschungsprojekte durchgeführt, welche die wichtigen ostalpinen Sulfidvorkommen auf ihre High-
Tech-Metalle untersuchen (Tab. 1). Folgende Ziele wurde mit den drei Projekten verfolgt:

1. Erweiterung der Geochemie- und Isotopen-Datenbasis von österreichischen Lagerstätten und Integration in existierende GBA-Datenbasen (IRIS).

2. Neue Diskriminierung der Erzvorkommen gemäß ihrer chemischen Charakteristika.

3. Entwicklung spezieller Genesemodelle der Buntmetallvorkommen im ÖSK und darauf aufbauend eines empirischen Modells, welches die Abhängigkeiten der HTMetalle von geologischen Faktoren beschreibt.

4. Neubewertung der Sicherungswürdigkeit der Buntmetall-Lagerstätten und Vorkommen hinsichtlich der Ressourcenpotentiale für HT-Metalle.

Über Ergebnisse der Leobner Projekte wurde bereits berichtet [5]. In diesem Beitrag werden die Ergebnisse der drei Projekte zusammenfassend dargestellt sowie für den Bereich des Ötztal-Stubai-Kristallins (ÖSK) exemplarisch auf ihre genetische Relevanz diskutiert (Masterarbeiten) ${ }^{1}$.

\subsection{Allgemeines zu Spurenelementen in Sulfiden}

Spurenelementanalysen in Sulfiderzen erleben derzeit eine Renaissance, da das wirtschaftliche Interesse an Sulfiden als potenzieller Wirt für HT-Metalle erheblich gestiegen ist. Das HT-Metall-Potenzial der einzelnen Minerale, die im metallurgischen Aufbereitungsprozess konzentriert werden können, kann durch in-situ Analysen erfasst werden [6]. Hier gilt die Laserablations-ICP-MS (LA-ICP-MS) als die bevorzugte Methode. Eine Reihe von in-situ LA-ICP-MS Arbeiten stellen die generelle Varianz der Spurengehalte in Sulfiden (namentlich Sphalerit, Chalkopyrit und Galenit) verschiedener Buntmetalllagerstättentypen heraus [7-10]. Sphalerit, und im Falle für In auch Chalkopyrit, stellen die

1 Volgger, A.; Gasteiger, P.: Geochemische und petrologische Untersuchungen in der $\mathrm{Pb}-\mathrm{Zn}$ Lagerstätte Schneeberg im Bereich des Poschhausstollens (Ridnaun, Südtirol), unveröffentlichte Masterarbeiten, Institut für Mineralogie und Petrographie, Innsbruck: Universität Innsbruck, 2019,216S 


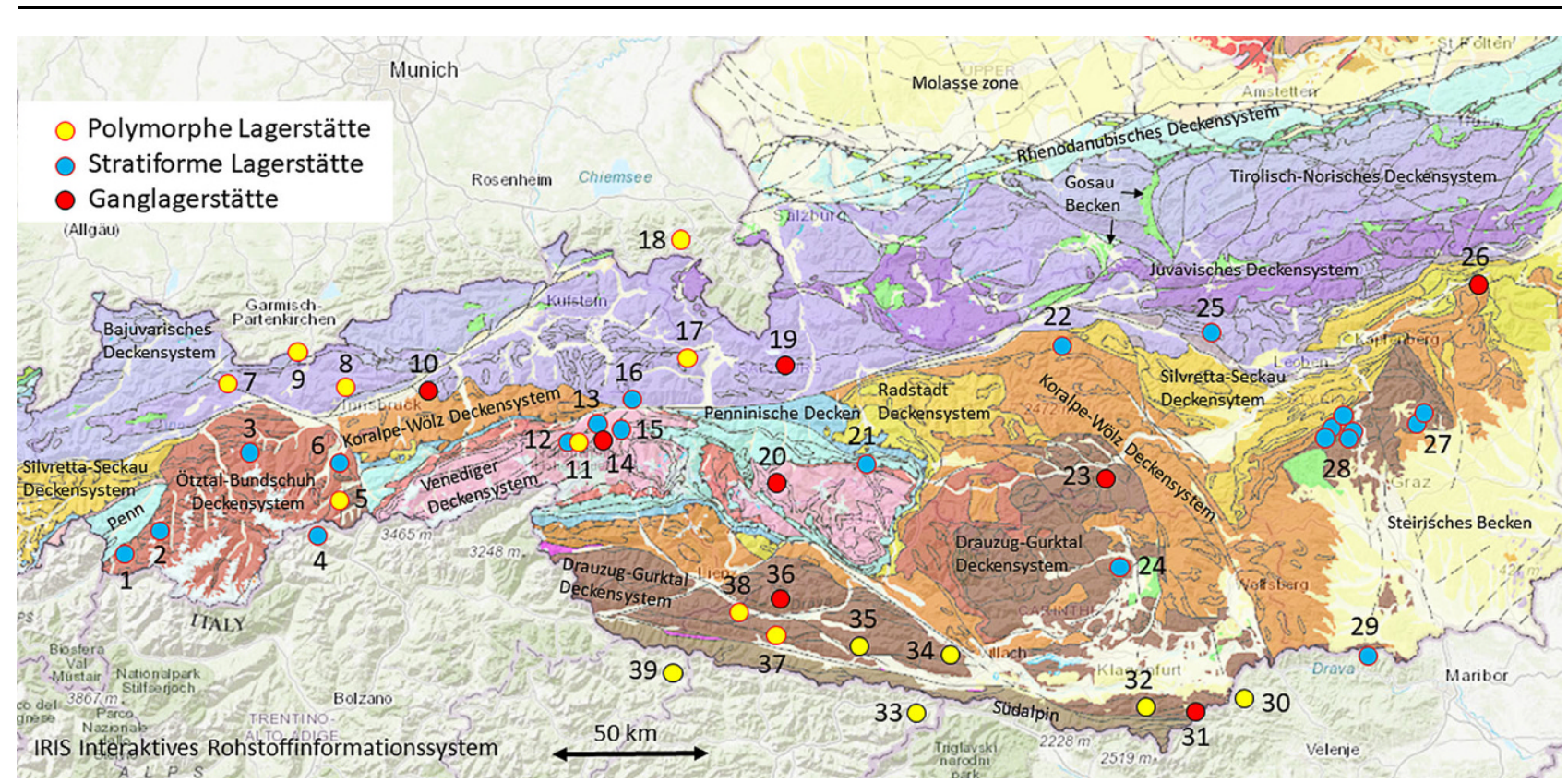

Abb. 1: Geologisch-tektonische Karte von Österreich mit Lage der untersuchten Vorkommen. Abkürzungen: $1=\mathrm{Nauders} \mathrm{(ÖSK} \mathrm{Pb-Zn);} 2=\mathrm{Tösens}($ ÖSK $\mathrm{Pb}-\mathrm{Zn}) ; 3=$ Wörgetal (ÖSK Cu-Fe); $4=$ Schneeberg (ÖSK Pb-Zn); $5=$ Obernberg $(\mathrm{Pb}-\mathrm{Zn}) ; 6=$ Serles $(\mathrm{Pb}-\mathrm{Zn}) ; 7=\mathrm{Silberleiten}(\mathrm{Pb}-\mathrm{Zn}) ; 8=\mathrm{Lafatsch}(\mathrm{Pb}-\mathrm{Zn})$; $9=$ Mittenwald $(\mathrm{Pb}-\mathrm{Zn}) ; 10=$ Bertagänge $(\mathrm{Cu}-\mathrm{Fe}) ; 11=$ Peitingalm $(\mathrm{Cu}) ; 12=$ Hochfeld $(\mathrm{Cu}) ; 13=$ Brenntal $(\mathrm{Cu}-\mathrm{Fe}) ; 14=\mathrm{Achselalpe}(\mathrm{Zn}-\mathrm{Pb}-\mathrm{F}) ; 15=\mathrm{Haidbach}$

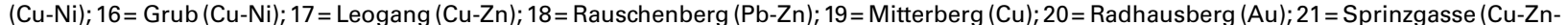

$\mathrm{Au}) ; 22=$ Walchen $(\mathrm{Cu}-\mathrm{Fe}) ; 23=$ Metnitz $(\mathrm{Pb}-\mathrm{Zn}) ; 24=$ Meiselding (Cu-Pb-Ag); $25=$ Kalwang $(\mathrm{Cu}) ; 26=$ Fröschnitzgraben $(\mathrm{Zn}-\mathrm{Pb}) ; 27=\mathrm{Arzberg}(\mathrm{Pb}-\mathrm{Zn}-\mathrm{Ag})$; $28=$ Grazer Paläozoikum westlich der Mur (Pb-Zn-Ag); $29=$ Remschenigg $(\mathrm{Cu}-\mathrm{Pb}-\mathrm{Zn}) ; 30=$ Mezica $(\mathrm{Pb}-\mathrm{Zn}) ; 31=\mathrm{Koprein}(\mathrm{Pb}-\mathrm{Zn}) ; 32=\mathrm{Hochobir}(\mathrm{Pb}-\mathrm{Zn})$; $33=$ Raibl $(\mathrm{Pb}-\mathrm{Zn}) ; 34=$ Bleiberg-Kreuth $(\mathrm{Pb}-\mathrm{Zn}) ; 35=$ Radnig $(\mathrm{Pb}-\mathrm{Zn}) ; 36=$ Drassnitz $(\mathrm{Pb}-\mathrm{Zn}-\mathrm{Ag}) ; 37=\mathrm{Jauken}(\mathrm{Pb}-\mathrm{Zn}) ; 38=\mathrm{Pirkach}(\mathrm{Pb}-\mathrm{Zn}) ; 39=\mathrm{Salafossa}$ (Pb-Zn). Kartenbasis: GBA, IRIS Rohstoffe

bei weitem wichtigsten Wirtsminerale für die HT-Metalle $\mathrm{Ga}, \mathrm{Ge}$ und In (aber auch Sn, Sb, Cd und TI) dar, während Galenit als eher unbedeutender Wirt erscheint, der allerdings typischerweise Ag- und Sb-angereichert ist. Nach Stand der Forschung unterliegt die relative Verteilung der meisten Spurenelemente zwischen co-genetischen Erzphasen keinen eindeutig vorhersagbaren Regeln [11]. Daraus folgt, dass die Prozesse der Spuren-Anreicherung in Erzphasen, wie z.B. syngenetisch- und epigenetisch-hydrothermale Mineralisation und metamorphe Überprägung, nur im Ansatz verstanden sind.

\section{3 Übersicht der ostalpinen Buntmetall- sulfidvorkommen}

In den prä-mesozoischen Abfolgen der Ostalpinen Decken [12-14] sind zahlreiche Lagerstättenprovinzen dokumentiert. Die Eisen-, Buntmetall- $(\mathrm{Cu}, \mathrm{Pb}, \mathrm{Zn})$ und Edelmetallvorkommen $(\mathrm{Au}, \mathrm{Ag})$ sind entweder syngenetisch, bereichsweise durch nachfolgende Metamorphose und metasomatische Ereignisse überprägt, oder gänzlich epigenetisch gebildet worden. Intrusion von sauren Schmelzen während einer variszischen orogenen Phase im Karbon führte regional zu granitgebundenen Lagerstätten. In den mesozoischen Decksedimenten sind ebenfalls syngenetische und epigenetische Mineralisationen erhalten. Die bedeutenden Siderit-Buntmetall, Magnesit und $\mathrm{Pb}-\mathrm{Zn}$ Mineralisationen sind an extensionsbedingte Fluidmobilisation während Trias und Jura gebunden. Während der kretazi- schen bis neogenen alpidischen Orogenese bildeten sich strukturgebundene Gang- und Verdrängungslagerstätten.

Als tektonisch liegendste Lagerstättenprovinz haben die Erze in den Subpenninischen Decken im Tauernfenster (Venedigerdecke) eine besondere Bedeutung: hier treten vor allem stratiforme $\mathrm{Cu}, \mathrm{Ni}, \mathrm{Pb}-\mathrm{Zn}$ sowie granitgebundene W- und Mo-Vererzungen auf (Abb. 1). Diese Deckeneinheiten werden von geringmächtigen mesozoischen Sedimenten überlagert und von mächtigen Ophiolith- und Flyschabfolgen der penninischen Decken überschoben. Diese führen zahlreiche Cu-Pyrit-Vererzungen, die als vulkanogene Massivsulfide interpretiert werden. Subpenninisches und Penninisches Deckensystem werden von den ostalpinen Decken überschoben, die sich tektonisch wiederum in unter- und oberostalpine Einheiten und zahlreiche Deckensysteme gliedern lassen. Die Kristalleinheiten sind vor allem im Bereich des ÖSK reich an Fe, $\mathrm{Cu}$ und $\mathrm{Pb}-\mathrm{Zn}$ Vererzungen. Die bedeutendsten Metallanreicherungen in den ostalpinen Decken sind an niedrig- bis mittelgradig metamorph überprägte paläozoische Gesteine in einem WestOst-streichenden Korridor zwischen Arlberg und Semmering gebunden. Diese früher als Grauwackenzone bezeichneten Einheiten sowie die stratigraphisch ähnlichen Gurktaldecken und das Grazer und Eisenkappeler Paläozoikum zeigen eine Vielzahl von Fe-Cu-Pb-Zn-Ag-Vorkommen. Die permomesozoischen Decksedimente führen bedeutende $\mathrm{Pb}-\mathrm{Zn}$ Lagerstätten in mitteltriassischen Karbonatsedimenten („Bleiberg Typ“/"Alpiner Typ“). Strukturgebundene Vererzungen innerhalb der penninischen und ostalpinen Deckensysteme sind vor allem reich an Fe (Waldenstein: 

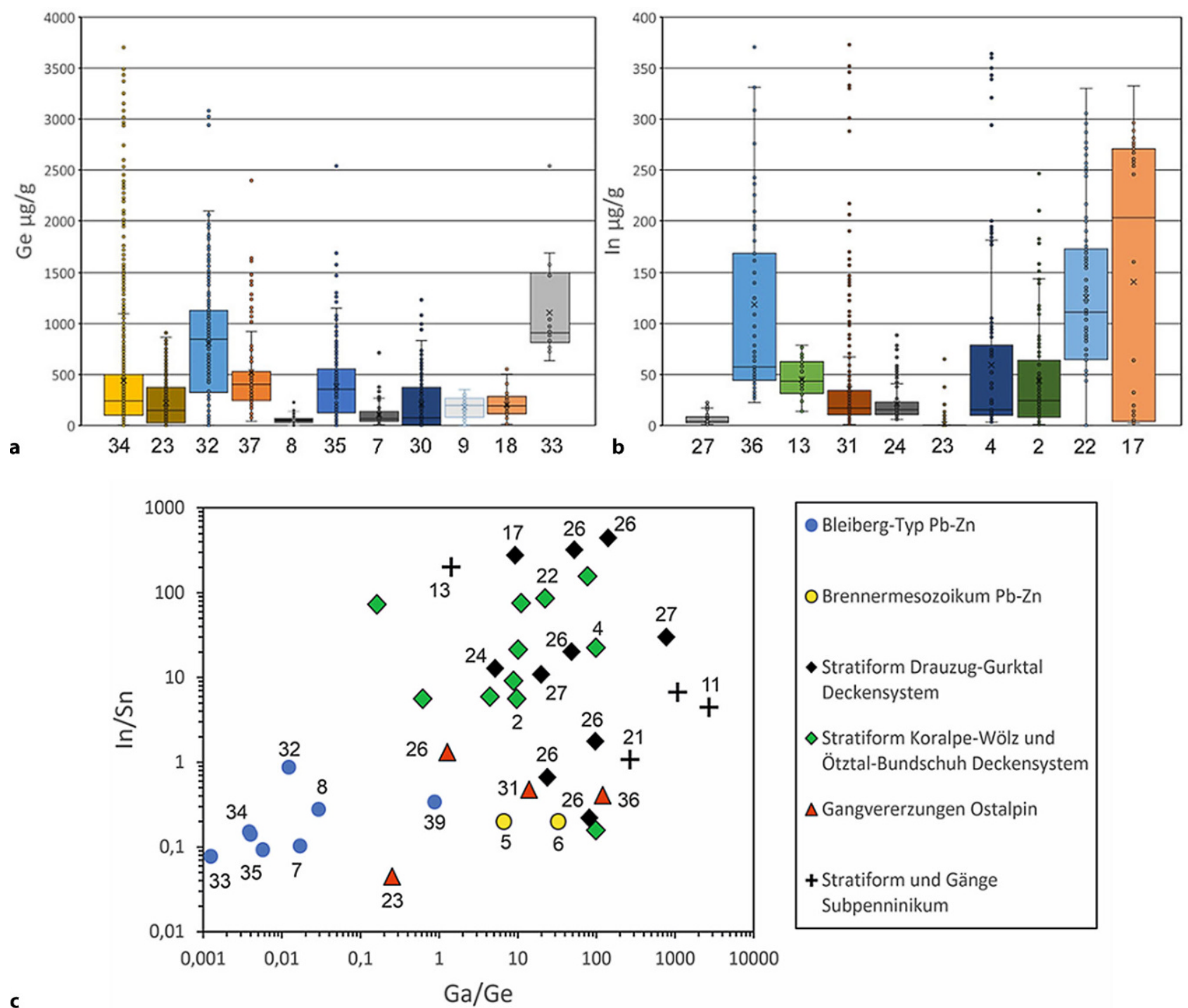

- Bleiberg-Typ Pb-Zn

O Brennermesozoikum Pb-Zn

- Stratiform Drauzug-Gurktal Deckensystem

$\diamond$ Stratiform Koralpe-Wölz und Ötztal-Bundschuh Deckensystem

$\Delta$ Gangvererzungen Ostalpin

+ Stratiform und Gänge

Subpenninikum

c

Abb. 2: Boxplotsfüra Geund b In in SphaleritverschiedenerVorkommen.c Ga/Ge und In/Sn aus Medianwerten aus SphaleritverschiedenerVorkommen in den Ostalpen. Nummern siehe Abb. 1

Hämatit; Hüttenberg: Siderit), Cu (Mitterberg) und Au (Tauerngold, Flatschach, Pusterwald).

\section{Probenherkunft und Untersuchungs- methoden}

Insgesamt wurden in den drei MRI-Projekten über 450 Proben aus mehr als 50 Vorkommen in den Ost- und Südalpen mittels LA-ICP-MS untersucht (Tab. 1). Dabei wurden 25 Neben- und Spurenelemente bestimmt. Der Fokus lag auf Sphalerit (6800 Messpunkte). Im zweiten MRI-Projekt wurden Chalkopyrit, Pyrit und Pyrrhotin aus $\mathrm{Pb}-\mathrm{Zn}, \mathrm{Cu}$ und einigen Au-Lagerstätten analysiert. Der Probensatz für das Innsbruck-Projekt umfasst 62 Stück und ist eine repräsentative Auswahl von 18 Vorkommen, darunter 13 aus der $\mathrm{Pb}$ $\mathrm{Zn}$ (mit drei untergeordneten $\mathrm{Cu}$-Fe Vorkommen) und 5 aus der Cu-Fe Domäne. Neben diesen Proben gibt es einen speziellen Satz aus dem Schneeberger Poschhausstollen (Mas- terarbeit Gasteiger \& Volgger 2018, Uni Innsbruck). Sphalerit, Chalkopyrit und Silikate wurden analysiert. Messungen der Gesteinsgeochemie, Kohlenstoff- und SauerstoffIsotopie, Strontium Isotopie, sowie Mikrochemie an Mikrosonde und Nano-SIMS komplettieren diese Detailuntersuchung des ÖSK.

\section{Spurenelementverteilungen in Sulfiden der Ostalpinen Vorkommen}

Die Ergebnisse belegen eine große Variabilität in der Zusammensetzung von Sphalerit, Chalkopyrit, Pyrit und Pyrrhotin. Selbst in genetisch verwandten Vorkommen variieren die Spurenelementkonzentrationen sehr stark. Zwei Sphalerittypen können klar abgegrenzt werden: [1] in nicht metamorphen Karbonatsedimenten sind die Sphalerite typischerweise arm an Fe $(<1 \%), \mathrm{Mn}, \mathrm{Co}, \mathrm{Ga}, \mathrm{In}, \mathrm{Sn}$ und Sb. Sie können jedoch zum Teil erhebliche Konzentratio- 
Abb. 3: a Statistische Darstellung der HT-Metalle in Sphalerit in Vorkommen des ÖSK. b Cd vs Mn/Co in Sphalerit. Der "Ostalpine Sphalerit-Median" (Median der Mediane aller Vorkommen) hat folgende Werte $($ in $\mu \mathrm{g} / \mathrm{g})$ : Mn 62, Fe 35010 Co 33, Ni 0,2, Cu 264, Ga 4,5, Ge 0,3 , As 0,7, Se 12, Ag 14, Cd 2184, In 1,7, Sn 0,37, Sb 3,5, TI $0,03, \mathrm{~Pb} 30$
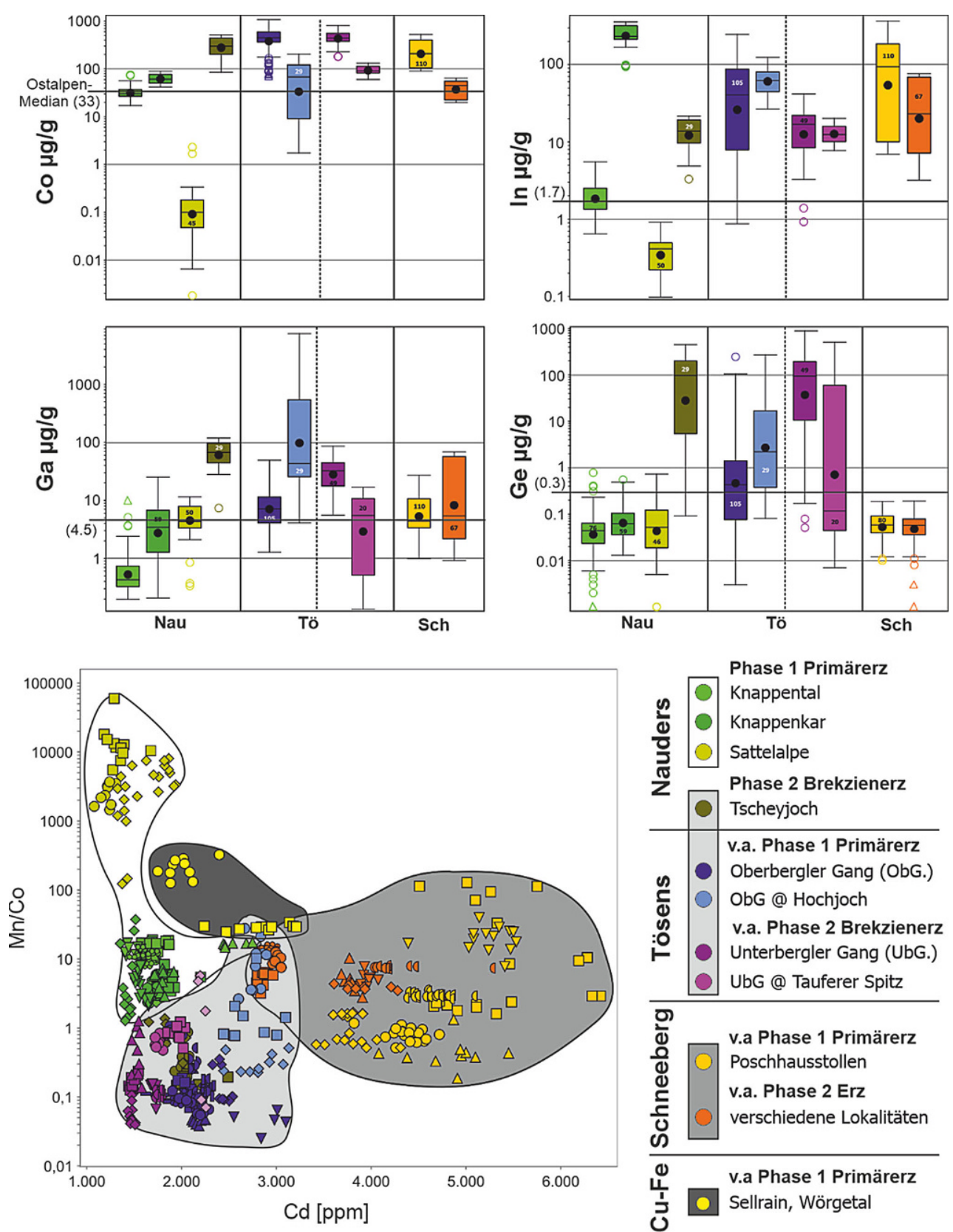

nen der Elemente Ge (bis $>500 \mu \mathrm{g} / \mathrm{g}$ ), As, TI und Pb aufweisen (Abb. 2a; [2]). In stratiformen und Ganglagerstätten in niedrig- bis mittelgradig metamorphen Gesteinen sind die Sphalerite angereichert an $\mathrm{Fe}, \mathrm{Co}, \mathrm{Ni}, \mathrm{Cu}, \mathrm{Ag}$, In und $\mathrm{Sn}$ (Abb. 2b). In dem Diagramm Ga/Ge versus In/Sn können diese Typen weitestgehend diskriminiert werden (Abb. 2c).

Die Sphalerite in der ladinischen und karnischen Stufe des Drauzug-Gurktal-Deckensystems (Typlagerstätte Bleiberg) und des Südalpins (Raibl, Salafossa, Mezica) sind recht ähnlich und unterscheiden sich im Wesentlichen in der Magnitude ihrer Elementanreicherungen. Vorkommen in gleichaltrigen Gesteinen der Nördlichen Kalkalpen (Tirolisch-Norisches Deckensystem) sind demgegenüber an $\mathrm{Ag}$ angereichert. Sphalerite in Triaskarbonaten des Brennermesozoikums (Ötztal-Bundschuh Deckensystem) sind auf- fallend arm an Spurenelementen. Die stratiformen Erzlager des Grazer Paläozoikums und der Gurktaldecke führen generell Fe-reichere Sphalerite (1-9\% Fe), die meist nur gering an $\mathrm{Co}, \mathrm{Ag}, \mathrm{Ga}$, Sb und In angereichert sind. In der polymetallischen Lagerstätte Walchen (Ennstaler Phyllitzone, Koralpe-Wölz Deckensystem) treten Fe-In-Co-Cu-reiche Sphalerite gemeinsam mit In- und Sn-reichem Chalkoyprit auf. Die stratiforme Lagerstätte Schneeberg im ÖSK (Südtirol) ist ebenfalls durch die Assoziation Fe-Co-Cu-In charakterisiert. Kleinere stratiforme und polymorphe Vorkommen im ÖSK sind Fe-reich und führen $\mathrm{Co}, \mathrm{In}, \mathrm{Ag}$ und $\mathrm{Hg}$ als wichtige Spurenelemente. Ganglagerstätten können erhöhte Konzentrationen aller Spurenmetalle führen, sind teilweise aber auch auffallend arm an HT-Metallen, z. B. Achselalm (Habachserie des Tauernfensters). 
Chalkopyrite aus 26 Vorkommen führen $\mathrm{Zn}, \mathrm{Ag}, \mathrm{Sn}$, Se, $\mathrm{Pb}$ und $\mathrm{Mn}$ als wichtigste Spurenelemente. Die Mediankonzentrationen von $\mathrm{Ag}$ und $\mathrm{Sn}$ können mehrere $100 \mu \mathrm{g} / \mathrm{g}$ bis einige Zehntelprozent erreichen. Die Ag-reichsten Chalkopyrite mit Medianwerten $>600 \mu \mathrm{g} / \mathrm{g}$ stammen aus den Vorkommen Meiselding und Drassnitz. Medianwerte für In erreichen in den Vorkommen Walchen und Drassnitz Werte $>40 \mu \mathrm{g} / \mathrm{g}$. Die Konzentrationen der kritischen Rohstoffe Co, $\mathrm{Ge}$ und $\mathrm{Ga}$ sind dagegen niedrig und übersteigen nur in Ausnahmen $10 \mu \mathrm{g} / \mathrm{g}$. Am Glücksgrat im Stubaital wurden ungewöhnlich hohe Se, In $(360 \mu \mathrm{g} / \mathrm{g})$, Sn und Ge-Konzentrationen ermittelt.

Pyrite aus 26 Vorkommen führen $\mathrm{Co}, \mathrm{Ni}$ und $\mathrm{As}$ als häufigste Spurenelemente, gefolgt von $\mathrm{Mn}, \mathrm{Pb}, \mathrm{Zn}$ und $\mathrm{Cu}$. Die Konzentrationen von In, $\mathrm{Ge}$ und $\mathrm{Ga}$ erreichen maximal $20 \mu \mathrm{g} / \mathrm{g}$. Die Co- und Ni-reichsten Pyrite wurden im Vorkommen Haidbach (Median $3754 \mu \mathrm{g} / \mathrm{g} \mathrm{Co}, 935 \mu \mathrm{g} / \mathrm{g} \mathrm{Ni}$ ) analysiert [15]. Co-Medianwerte in Pyrit aus Vorkommen im Grazer Paläozoikum, Walchen und Mitterberg liegen bei etwa $500 \mu \mathrm{g} / \mathrm{g}$, jene aus vielen Ganglagerstätten und karbonatgebundenen $\mathrm{Pb}-\mathrm{Zn}$ Erzen deutlich darunter.

Pyrrhotin wurde in lediglich in 6 Vorkommen analysiert. Hier sind $\mathrm{Co}, \mathrm{Ni}$ und $\mathrm{Mn}$ die einzigen relevanten Spurenelemente. In der Hälfte der Vorkommen ist Ni gegenüber Co dominant (Median $5755 \mu \mathrm{g} / \mathrm{g} \mathrm{Ni}$, Haidbach), während in Leogang, Walchen und Drassnitz Co überwiegt (Medi- an $250 \mu \mathrm{g} / \mathrm{g}$ Co, Leogang). Konzentrationen von $\mathrm{Ge}, \mathrm{Ga}$ und In liegen meist deutlich unter $1 \mu \mathrm{g} / \mathrm{g}$

Eine statistische Übersicht für das ÖSK zeigt die heterogene Verteilung der Vorkommen (Abb. 3a). Das Diagramm Cd gegen Mn/Co (Abb. 3b) wurde entwickelt, um eine möglichst komplette Diskriminierung der Vorkommen anhand der Sphaleritchemie zu erzielen. Das Diskriminierungsdiagramm Sn versus In zeigt eine recht gute Abtrennung der Vorkommen (Abb. 3c).

\section{Sulfidmineralisationen des ÖSK im geologischen Kontext}

Der polymetallische $(\mathrm{Cu}-\mathrm{Fe}-\mathrm{Zn}-\mathrm{Pb} \pm \mathrm{Ag} \pm \mathrm{Bi} \pm \mathrm{As} \pm \mathrm{Ni} \pm \mathrm{Co} \pm \mathrm{Sb} \pm$ Au) Erzbezirk Ötztal-Stubai (z. B. Tösens, Nauders, Schneeberg) ist von weitestgehend stratiformen Sulfidvererzungen in spät-präkambrischen/paläozoischen Paragneisen, lokal auch in Karbonaten und Amphiboliten, gekennzeichnet (Abb. 4). Zwei regional getrennte Erzmetall-Domänen sind markant: eine $\mathrm{Cu}-\mathrm{Fe}$ dominierte im Norden und eine $\mathrm{Pb}-\mathrm{Zn}$ dominierte im Süden [16]. Ob das ÖSK eine beckensystematische Einheit darstellt oder sich aus mehreren Teilen zusammenfügte, ist noch ungeklärt. Die BuntmetallLagerstätten sind als submarin-exhalative "SEDEX“ Lagerstätten zu deuten, während assoziierte Gangerze syn-
Abb. 4: Geologisch-tektonische Karte von Ötztal-StubaiKristallin (nach [32]), mit metamorphen Zonengrenzen [33] und Lokalitäten der $\mathrm{Zn}-\mathrm{Pb}$ und Cu-Fe Vorkommen (nach [16])

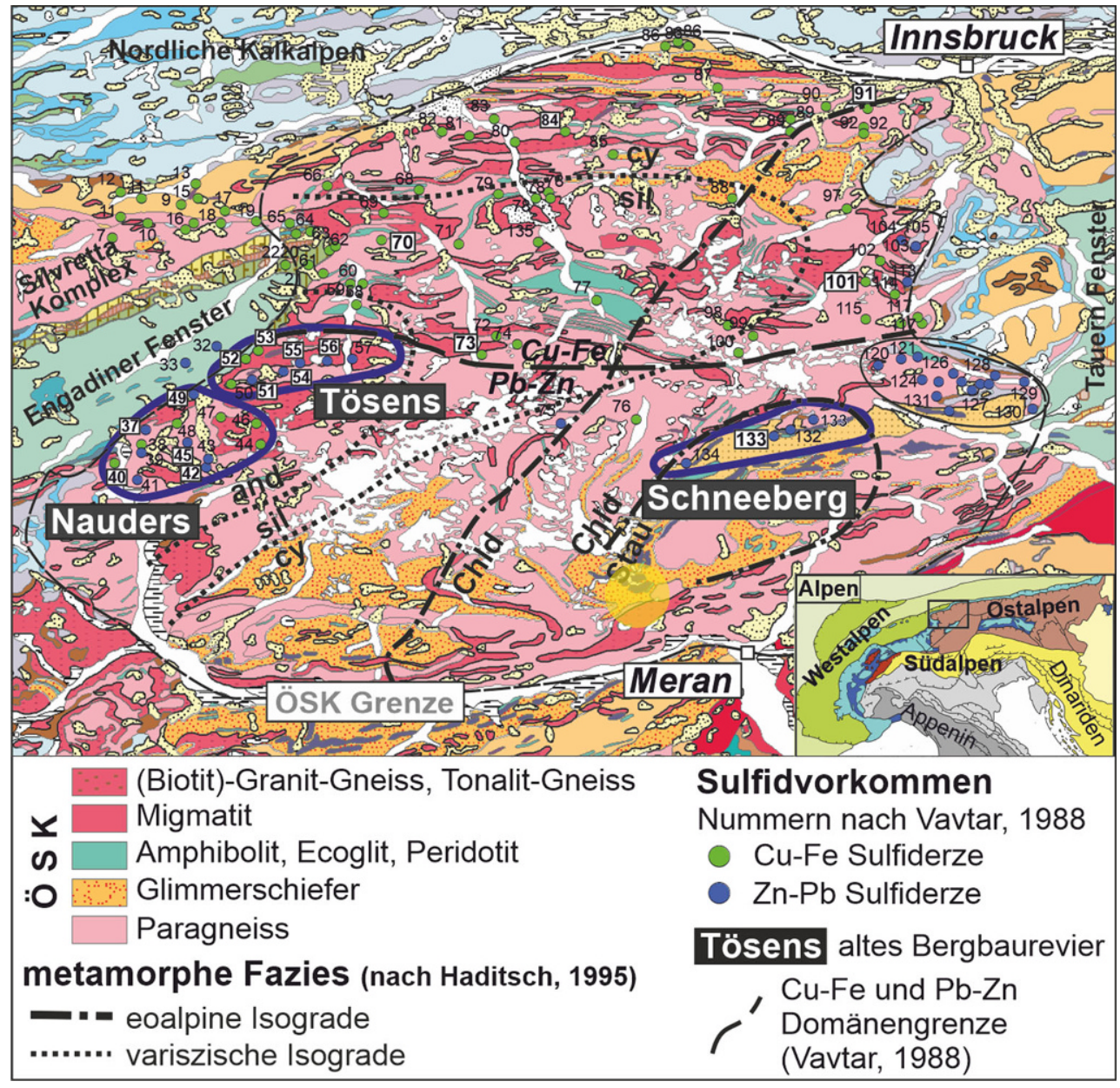


Abb. 5: a Paragenetische Sequenz; $\mathbf{b}-\mathbf{g}$ Petrographie der Erztypen im ÖSK
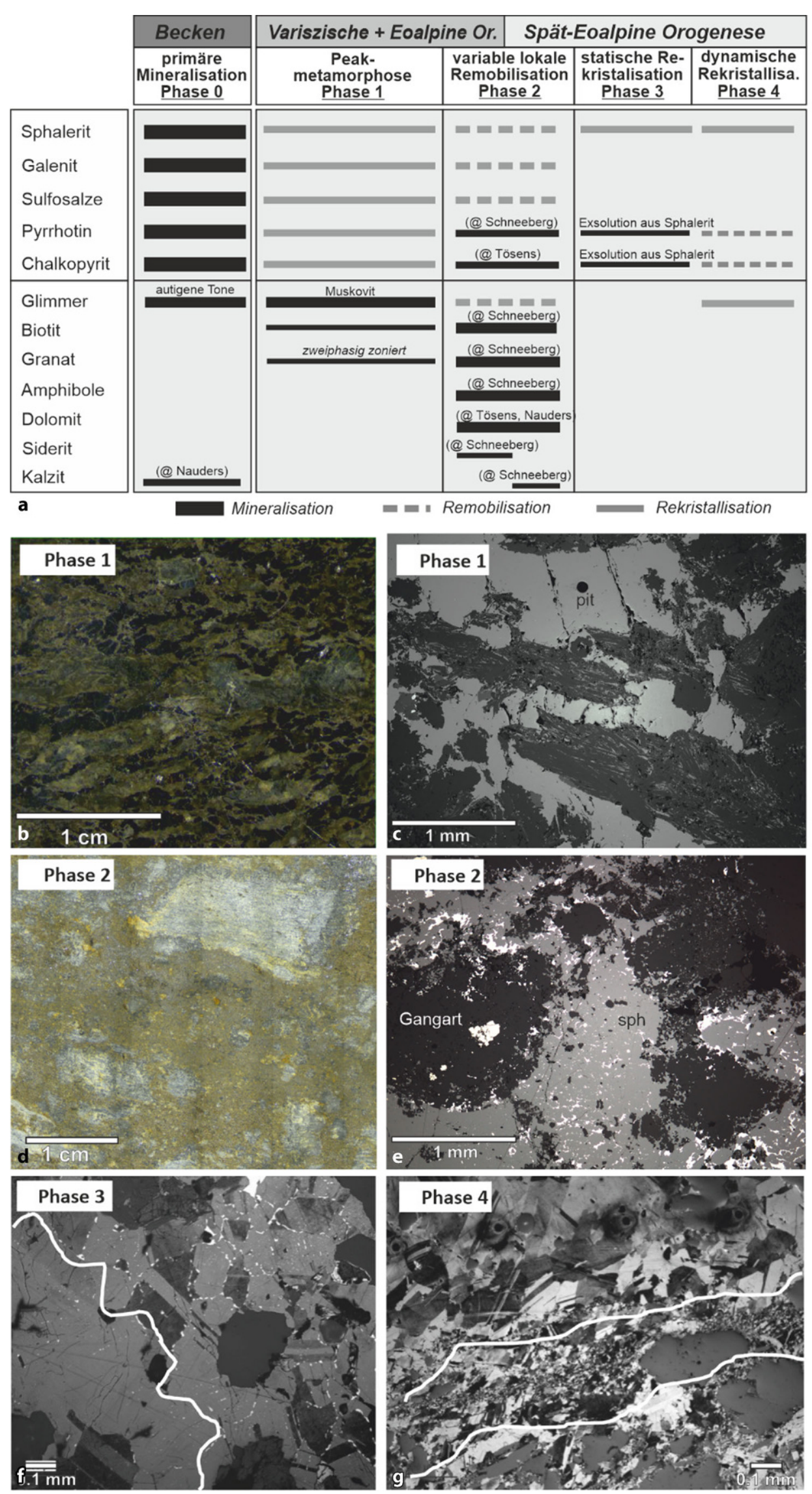
metamorphe Remobilisate darstellen [16]. Die Metalle in SEDEX Systemen stammen aus der liegenden Beckenstratigraphie und wurden durch zirkulierende Fluide (erhitztes Meerwasser und Solen aus den Sedimenten) mobilisiert [17-21]. Im ÖSK ist die Metallherkunft aus der umgebenden Lithostratigraphie durch Pb-Isotopendaten bekräftigt worden $[2,22,23]$. Die Einarbeitung in die variszische und eoalpidische Orogenese führte zu einem strukturell komplexen Deckenbau mit niedrig- bis hochtemperatur- und lokal hochdruckbetonten metamorphen Überprägungen [24-30]. Der prägende Metamorphosegrad nimmt generell nach Südosten zu, mit höchstfazieller eoalpidischer Ausprägung im Schneeberg-Revier. Im Westen (Tösens, Nauders) ist die variszische amphibolitfazielle Metamorphose grünschieferfaziell retrograd überprägt worden.

Die generell fünf-phasigen Entwicklungen der Erze in den Revieren des ÖSK sind in Abb. 5a zusammengefasst. Feinlaminierte Sulfid-führende Glimmerschiefer und Sphalerit-Phyllonite sind als Relikt einer synsedimentären Ablagerung (Phase 0 der Entwicklung), wahrscheinlich als tonreiche Sulfidschlämme, zu sehen. Primäre Sulfidphasen sind durch Überprägung nicht mehr vorhanden, früheste erhaltene Sulfide werden der Peakmetamorphose zugeordnet (Phase 1, Abb. 5b, c). Diese Überprägung fand entweder während der variszischen Orogenese (Nauders, Tösens) oder im Fall von Schneeberg der eoalpidischen Orogenese statt. Die Phase 2 ist eine Sequenz aus deformationskontrollierter Remobilisation der Primärerze, die in Tösens und Nauders als hydrothermale Gang- und Brekzienvererzung (Abb. 5d,e) und in Schneeberg als lagige Intraremobilisation ausgeprägt ist. Karbonatisierung ist eine typische Begleiterscheinung solcher Remobilisate. Es ist anzunehmen, dass sich im Zuge orogener Spröd-Deformationen druckgetriebene Formations- oder metamorphe Wässer durch die obere Kruste bewegten und Sulfidphasen remobilisierten. Diese wurde in Folge wieder metamorphisiert: Phase 3 und 4 sind niedrigradige/retrograde metamorphe Überprägungen mit statischer Textur-Equilibrierung und dynamischer Rekristallisation in Scherzonen (Abb. 5f,g). Diese Verformung samt Metasomatose wird als spätere eoalpidische Phase interpretiert. Da Pyrrhotin stets als Einschlüsse bzw. Klasten im Rekristallisat vorkommt, sind hier Temperaturen von maximal $300^{\circ} \mathrm{C}$ anzunehmen [31].

\section{Faktoren der Spurenelementverteilung in Sulfiden des ÖSK}

Die Studie im ÖSK ergab eine Reihe geologischer und mineralogisch-geochemischer Eigenschaften (Faktoren), die charakteristisch für Domänen, Reviere, Vorkommen und Proben sind. Aufgrund dessen wurde ein Vergleich der Sphalerit- und Chalkopyrit-Chemie durchgeführt, um die Signifikanz jener Faktoren zu evaluieren.

\subsection{Faktor Becken-Tektonik, Magmatismus, Lithostratigraphie}

Auf dem regionalen Maßstab ist zwischen der südlichen $\mathrm{Pb}-\mathrm{Zn}$ und nördlichen $\mathrm{Cu}-\mathrm{Fe}$ metallogenen Domäne zu unterscheiden. Die regionale Variation des sedimentär-magmatischen Ablagerungsraums hat einen direkten Einfluss auf die Metallisation und Sphalerit-Chemie. Es zeigt sich, dass Sphalerite in der Cu-Fe Domäne reicher an $\mathrm{Cu}, \mathrm{Mn}$, $\mathrm{Ag}, \mathrm{Bi}, \mathrm{As}$ und ärmer an $\mathrm{Co}, \mathrm{Ni}, \mathrm{Hg}$ sind. Im Falle von $\mathrm{Cu}$ ist die Anreicherung sicher auf den Überschuss im mineralisierenden Fluid zurückzuführen. Die Anreicherungen von $\mathrm{Ag}, \mathrm{Bi}$ und As sind vom Cu-Gehalt abhängig. Erhöhtes $\mathrm{Mn}$ zeigt allgemein keine Korrelation zu $\mathrm{Cu}$ und ist daher eine unabhängige Signatur der $\mathrm{Cu}-\mathrm{Fe}$-Domäne. Andererseits sind $\mathrm{Hg}$, Co und Ni Signaturen der Pb-Zn-Domänen.

\subsection{Faktor lokales Ablagerungsmillieu (Lithostra- tigraphie, Vent-Proximalität, Vererzungstyp)}

Die Nähe zum Exhalationsherd wird für viele SEDEX Lagerstätten als einer der wichtigen Faktoren erachtet, der Chemismus und Mineralogie der Erze beeinflusst [20]. Daneben haben auch Lithostratigraphie und Vererzungstyp/-Paragenese enormen Einfluss auf die lokale Sulfidchemie.

Einige Vorkommen in der südlichen $\mathrm{Pb}-\mathrm{Zn}$ Domäne sind mit Amphiboliten assoziiert, und diese Vorkommen führen typischerweise $\mathrm{Cu}-\mathrm{Fe} \pm \mathrm{Zn}$ Erze. Anhand des Chalkopyrits lassen sich Aussagen treffen, in wie weit sich das lokale Vorhandensein von Amphiboliten auf die Spuren in Sulfiden auswirkt. Der Vergleich zeigt höhere Co, Bi, Se Werte in Chalkopyriten in $\mathrm{Cu}-\mathrm{Fe} \pm \mathrm{Zn}$ Erzen gegenüber höheren $\mathrm{Ga}$, $\mathrm{Ge}, \mathrm{Pb}, \mathrm{Ag}, \mathrm{Sb}, \mathrm{Sn}$ Werten in solchen der $\mathrm{Pb}-\mathrm{Zn} \pm \mathrm{Cu}$ Erze. Die letztere Element-Gruppe ist mit Sulfosalzen assoziiert, woraus sich rückschließen lässt, dass die Spurenelement-Zusammensetzung der Chalkopyrite vom Gesamtchemismus der Erzparagenese abhängt. Andererseits sind die Elemente $\mathrm{Co}, \mathrm{Bi}$ und Se Indikatoren für Amphibolite in den Umgebungsgesteinen. Mit Sphalerit ließ sich diesbezüglich keine Aussage machen, da die $\mathrm{Cu}-\mathrm{Fe} \pm \mathrm{Zn}$ Erze kaum messbaren Sphalerit führten.

Metallverhältnisse der Gesamtgesteinsgeochemie sind Indizien für die Nähe zum Exhalationsherd, d.h. Fluid-, Stoff- und Hitzequelle [20]. Im ÖSK ließen sich anhand der Gesamtgesteinsgeochemie auf regionalem und lokalem Maßstab recht klare Trends bzgl. einer solchen Proximalität herleiten (Abb. 6). Auf regionalem Maßstab ist Fe in Sphalerit ein Indikator für Proximalität zum Vent (vergleichbar mit Ergebnissen von [34]). Eine Abreicherung von $\mathrm{Cu}, \mathrm{As}$, und $\mathrm{Bi}$ in proximalen Sphaleriten ist wahrscheinlich eine Folge der bevorzugten Partitionierung in paragenetisch assoziierten Chalkopyrit $[20,21]$. Auf lokalem Maßstab im Schneeberger Poschhausstollen sind diese Proxies nicht eindeutig ausgeprägt. Hier zeigen sich aber andere Trends: $\mathrm{Cd}, \mathrm{Hg}, \mathrm{Pb}, \mathrm{Mn}, \mathrm{As}, \mathrm{Sb}, \mathrm{Cr}$ im Sphalerit steigen mit Nähe zum prognostizierten Exhalationsherd an, während Co, In, $\mathrm{Ga}, \mathrm{Sn}, \mathrm{Bi}$ abfallen. Erhöhte $\mathrm{Cd}$ und Fe Werte werden von höheren Temperaturen kontrolliert, während $\mathrm{Pb}, \mathrm{Hg}, \mathrm{As}, \mathrm{Sb}$ mit Sulfosalz-Einschlüssen einher gehen. Die relative $A b$ - 


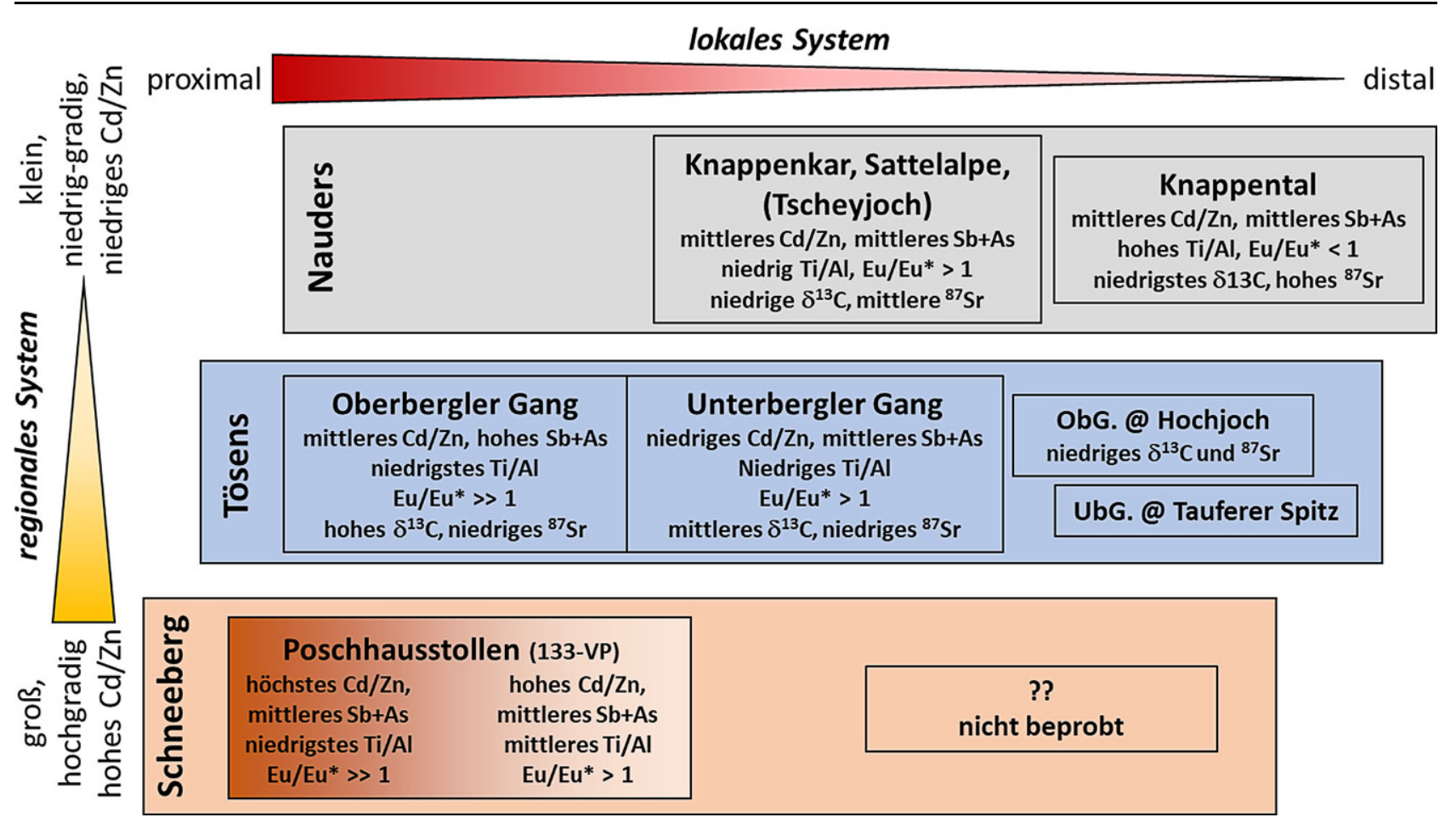

Abb. 6: Synthese der räumlich-temporalen Ausprägung des Mineralsystems im ÖSK, basierend auf Lagerstättengröße sowie Indikatoren der Gesamtgesteins-Geochemie (Dank an G. Hobiger, Geologische Bundesanstalt Wien), Karbonat-Isotopie (Dank an C. Spötl, Geologisches Institut, Universität Innsbruck) und Strontium-Isotopie (Dank an M. Regelous, Universität Erlangen)

reicherung von Co, In, ( $\mathrm{Ga})$ mit Nähe zum Exhalationsherd erscheint konsistent auf regionalem und lokalem Maßstab.

\subsection{Faktor hydrothermale Erz-Remobilisation}

In Schneeberg zeigt sich, dass in den Remobilisaten Cd, $\mathrm{Hg}, \mathrm{Mn}, \mathrm{Pb}, \mathrm{Ag}, \mathrm{Sb}, \mathrm{As}$ ansteigen und $\mathrm{Co}, \mathrm{In}, \mathrm{Ga}, \mathrm{Ge}, \mathrm{Sn}$, $\mathrm{Bi}$ abfallen. Die typischen Sulfosalzmetalle Ag, Sb, As sind deswegen erhöht, da diese Erze weniger Sulfosalz führen, und die Metalle vom Sphalerit (und Galenit) aufgenommen werden. In den Remobilisat-Gängen im Tösens Oberbergler Gang sind $\mathrm{Cu}$, In, $\mathrm{Ga}$, Ge, erhöht und nur Bi signifikant verringert. Die Brekzienvererzungen in Tösens Unterbergler Gang verhalten sich ähnlich, wie auch die Nauders Brekzienerze, mit Ausnahme des stark angereicherten As. Ähnlich zu Schneeberg ist ein niedriges Ag/Bi Verhältnis in Tösens und Nauders ein Proxy für Remobilisat-Erze. Das unterschiedliche Verhalten von $\mathrm{Cu}, \mathrm{In}, \mathrm{Ga}, \mathrm{Ge}$ belegt jedoch unterschiedliche Metallbudgets der Remobilisation in den Lokalitäten, die am ehesten auf Umgebungsgesteine (z. B. Cu und In aus den Diabasgängen in Tösens) zurückzuführen sind.

In den Chalkopyrit-reichen Gangerzen wirkt sich die Partitionierung unter den co-genetischen Phasen auf die Sphaleritchemie aus: Es zeigt sich, dass Chalkopyrit der präferierte Wirt für $\mathrm{Sn}, \mathrm{Ge}, \mathrm{Ag}$ und $\mathrm{Bi}$ ist.

\subsection{Faktor prograde Metamorphose (Rekristalli- sation)}

Obwohl prä-Alpine und eoalpine Metamorphosen systematisch auf dem regionalen Maßstab variieren, ist nur eine qualitative Bewertung der Zusammenhänge zwischen Metamorphose und Sulfidchemie möglich - hier sind andere Faktoren relevant. An metamorphen hydrothermalen Erzparagenesen, die mineralogisch vergleichbar sind mit den Tösens und Nauders Gangerzen, wurde gezeigt, dass mit zunehmender metamorpher Umwandlung $\mathrm{Sn}, \mathrm{Ga}$ und In in co-genetischen Chalkopyrit partitionieren [11]. Dies kann mit Daten des ÖSK bestätigt werden. Bemerkenswert ist das zuvor nicht beschriebene Verhalten von Ge, das auch in Chalkopyrit partitioniert. Sulfid-Kassiterit \pm Stannit Paragensen in Tösens und Glücksgrat [35] deuten auf einen Ausschluss von Sn aus der Struktur des peakmetamorphen Sphalerits hin. Wenngleich mineralogische Umwandlungen der prograden Metamorphose Einfluss auf die MetallPartitionierung haben, ändern diese das Budget jedoch nicht entscheidend.

\subsection{Faktor retrograde Rekristallisationen und Fluid-assistierte Deformation}

Die Mobilisierung von $\mathrm{Fe}$ und $\mathrm{Cu}$ ist von metamorpher Fazies, fluider Phase, und Deformation-Typus abhängig. Die in Schneeberg und Tösens häufigen Cu-Sulfid-Girlanden entlang statisch-equilibrierter Korngrenzen zeigen, dass die höheren $\mathrm{P} / \mathrm{T}$ Bedingungen dort eine ortsgebundene 

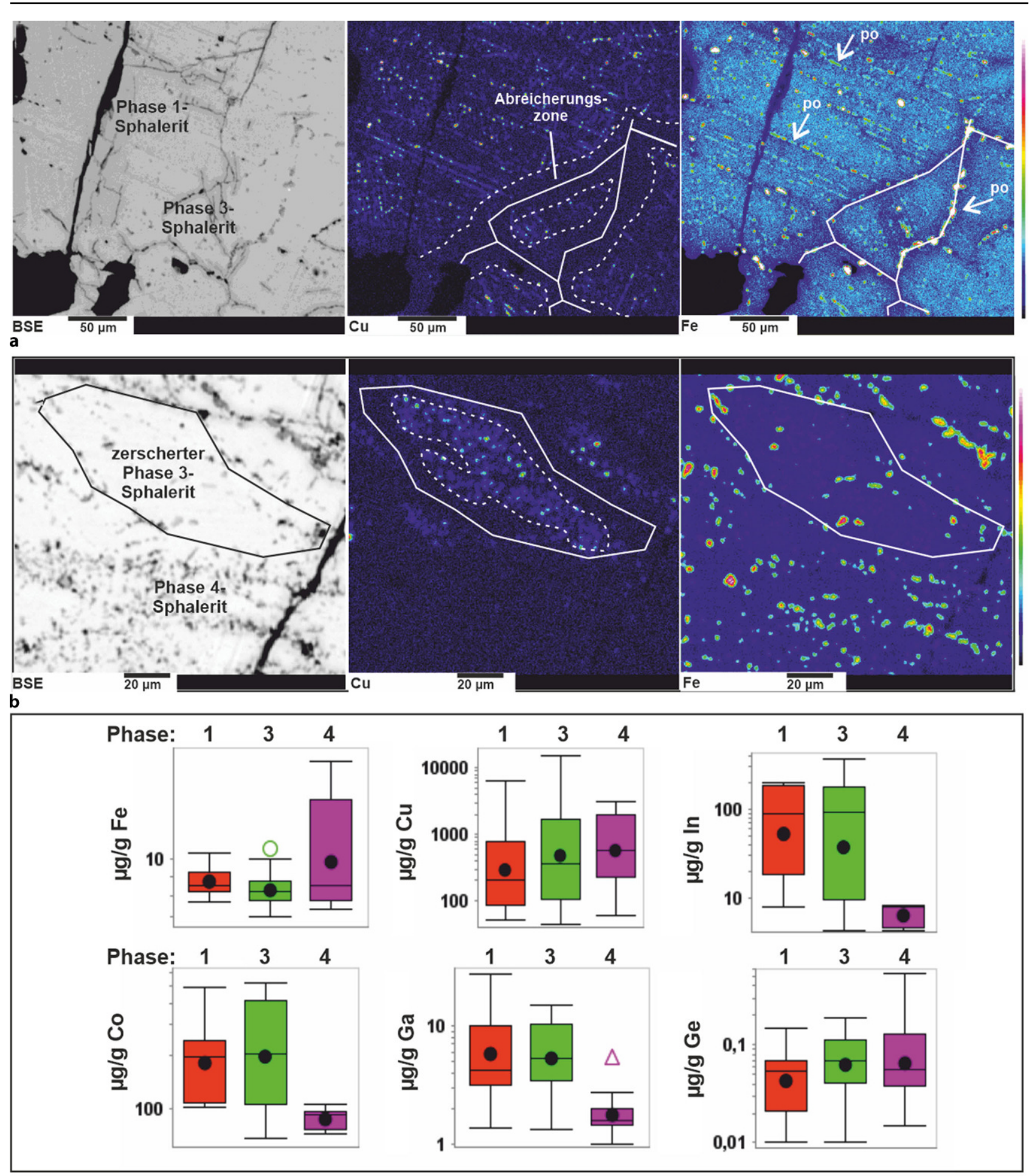

Abb. 7: Rasterelektonen-Rückstreubilder und Mikrosonden-Elementverteilungskarten für Fe und Cu von Sphalerit-Texturen aus a Tösens und b Nauders. c Box Plots zeigen die Chemismen der Sphalerit-Deformationstypen auf

Ausfällung von verschiedenen Cu-Sulfiden ermöglichten (Abb. 7a). In niedrig-gradigen Nauders Erzen führte dynamische Rekristallisation bei höherem Fluidfluss zu einem signifikanten Abtrag von $\mathrm{Cu}$ (Abb. 7b). Generell sind Diffusion und Fluid-gestützter Transport entlang von Korngrenzen wichtig. Bei statischer Rekristallisation erfährt nur $\mathrm{Hg}$ einen signifikanten Abfall, die dynamische Rekristallisation geht allerdings einher mit signifikantem Abfall an $\mathrm{Hg}$, In, $\mathrm{Co}$, und $\mathrm{Ga}$ und Anstieg an $\mathrm{Fe}, \mathrm{Cu}, \mathrm{Ge}, \mathrm{Ag}, \mathrm{Sb}$ (Abb. 7c). Für diesen selektiven Anstieg im Rekristallisat könnten fein verteilte Sulfosalzkörner verantwortlich sein, welche 
durch Mylonitisierung von ursprünglich gröberen Körnern hervorgerufen wurden.

\section{Forschungsfazit und Aussicht}

Unter Nutzung der umfangreichen Datensätze können Aussagen über die Präferenz von Spurenelementen in $\mathrm{Fe}-\mathrm{Cu}$ Zn-Sulfidparagenesen getroffen werden. Die Elemente Mn, $\mathrm{Ga}$, Se und Cd werden präferentiell in Sphalerit eingebaut; Co, Ni in Pyrit und Pyrrhotin; As in Pyrit; Ag und Sn im Chalkopyrit. Germanium wird in Sphalerit (karbonatgebundene $\mathrm{Pb}-\mathrm{Zn}$ Erze) und/oder Chalkopyrit eingebaut (Cu-reiche Erze), selten in Pyrit. Indium ist gleichfalls entweder an Sphalerit oder an Chalkopyrit gebunden. Antimon, TI, $\mathrm{Bi}$, Mo können in allen beteiligten Sulfidphasen eingebaut werden. Die Temperaturabhängigkeit der Sphaleritzusammensetzung wurde genutzt, um systematische Trends in der Zusammensetzung von Begleitsulfiden zu testen. Es scheint eine Temperaturabhängigkeit der Sn/Ag und Cd/ZnVerhältnisse im Chalkopyrit zu bestehen, die durch weitere Daten und experimentelle Untersuchungen überprüft werden sollte.

Der erbrachte Nachweis von komplexen, syngenetischen Verteilungsabhängigkeiten sowie metamorph-metasomatischen Remobilisierungs-Prozessen seltener Metalle in Sphalerit-reichen Erzen hat Auswirkungen auf die genetischen Untersuchungen von Erzen. Das gute Verständnis kann auch die metallurgische Aufbereitung von Spurenmetallen aus Sulfidkonzentraten verbessern. Die Faktoren lithostratographischer Kontext, Proximalität zum Exhalationszentrum, und metamorph-metasomatische Remobilisation und Partitionierung sind entscheidend. Metamorphe Überprägung hat keinen nennenswerten Effekt auf die Zn$\mathrm{Pb}$ Höffigkeit eines Vorkommens und eher geringe Auswirkungen auf die HT-Metall-Partitionierung.

Nach der Durchführung dreier MRI Projekte zum Thema Sulfidchemie sind weiterhin Fragen offen, die in Folgeprojekten bearbeitet werden sollten: physicochemische Faktoren, die den Einbau von Metallen in Sulfiden und co-genetischer Gangart (Silikate) bestimmen, sind ungeklärt und können durch experimentelle Untersuchungen entschlüsselt werden. Die Aufarbeitung der primären Beckenstratigraphie des ÖSK ist, gerade im Hinblick auf die variable Metallogenese, ein nicht nur regionalgeologisch interessanter Forschungsaspekt. Und nicht zuletzt warten die magmatogenen Vorkommen des Südalpins auf eine eingehende Untersuchung ihres HT-Metall-Potentials.

\section{7. Ökonomisches Fazit}

Eine wirtschaftliche Bewertung der untersuchten Vorkommen scheitert an fehlenden oder verifizierbaren Reservedaten. Ein Bewertungsversuch auf der Basis von Durchschnitts-Erzzusammensetzungen und derzeitigen Marktpreisen ergibt Metallinhalte zwischen 100 und $600 € /$ Tonne Erz, die zum großen Teil auf $Z n$ und $C$ u zurückzuführen sind. In karbonatgebundenen $\mathrm{Pb}-\mathrm{Zn}$ Vorkommen vom BleibergTyp können 10-30\% des Gesamterzwertes an Ge gebunden sein. Kobalt, Ag und In tragen nur maximal $5 \%$ (Co, Ag) bzw. $1 \%$ (In) des Metallwertes bei; Gallium spielt keine Rolle. In den großen Lagerstätten des ÖSK sieht die Situation derart aus, dass in Schneeberg In den Löwenanteil an den HAT-Metallen ausmachen würde $(75 \%)$, gefolgt von $\mathrm{Ga}(23 \%)$; in Tösens (Unterbergler Gang) wäre wiederum $\mathrm{Ge}(69 \%)$ wichtig, gefolgt von $\mathrm{Ga}(17 \%)$ und $\ln (11 \%)$. Aus geometallurgischer Sicht sind die gewonnenen Erkenntnisse zur Partitionierung in Sulfiden und metamorphen Mineralphasen aufschlussreich.

Funding. Open access funding provided by University of Innsbruck and Medical University of Innsbruck.

Open Access Dieser Artikel wird unter der Creative Commons Namensnennung 4.0 International Lizenz veröffentlicht, welche die Nutzung, Vervielfältigung, Bearbeitung, Verbreitung und Wiedergabe in jeglichem Medium und Format erlaubt, sofern Sie den/die ursprünglichen Autor(en) und die Quelle ordnungsgemäß nennen, einen Link zur Creative Commons Lizenz beifügen und angeben, ob Änderungen vorgenommen wurden.

Die in diesem Artikel enthaltenen Bilder und sonstiges Drittmaterial unterliegen ebenfalls der genannten Creative Commons Lizenz, sofern sich aus der Abbildungslegende nichts anderes ergibt. Sofern das betreffende Material nicht unter der genannten Creative Commons Lizenz steht und die betreffende Handlung nicht nach gesetzlichen Vorschriften erlaubt ist, ist für die oben aufgeführten Weiterverwendungen des Materials die Einwilligung des jeweiligen Rechteinhabers einzuholen.

Weitere Details zur Lizenz entnehmen Sie bitte der Lizenzinformation auf http://creativecommons.org/licenses/by/4.0/deed.de.

\section{Literatur}

1. Weber, L.: Der Österreichische Rohstoffplan - Archiv für Lagerstättenforschung, 26, Geologische Bundesanstalt, 26 (2012) S. 1-264

2. Weber, L.: Handbuch der Lagerstätten der Erze, Industrieminerale und Energierohstoffe Österreichs, Archiv für Lagerstättenforschung Geologische Bundesanstalt, 19 (1997) S. 1-607

3. Schroll, E.: über das Vorkommen einiger Spurenmetalle in BleiZink-Erzen der ostalpinen Metallprovinz, Tschermaks mineralogische und petrographische Mitteilungen, 5 (1955) S. 183-208

4. Henjes-Kunst, E.: The Pb-Zn Deposits in the Drau Range (Eastern Alps, Austria/Slovenia): A Multianalytical Research Approach for Investigation of the Ore-Forming Mechanisms, Doktorarbeit, Lehrstuhl für Rohstoffmineralogie, Leoben: Montanuniversität, 2014

5. Melcher, F; Onuk, P.: Potential of critical high-technology metals in eastern alpine base metal sulfide ores, BHM Berg-und Hüttenmännische Monatshefte, 164 (2019), S. 71-76

6. Cook, N.J., et al.: Trace Element Distributions in Sulphides: Progress, Problems and Perspectives, Acta Geologica Sinica - English Edition, 88 (2014), S. 1444-1446

7. Cook, N.J., et al.: Trace and minor elements in sphalerite: A LAICPMS study, Geochimica et Cosmochimica Acta, 73 (2009), S. 4761-4791

8. George, L., et al.: Trace and minor elements in galena: A reconnaissance LA-ICP-MS study, American Mineralogist, 100 (2015), S. 548-569

9. Ye, L., et al.: Trace and minor elements in sphalerite from base metal deposits in South China: a LA-ICPMS study, Ore Geology Reviews, 39 (2011), S. 188-217

10. Frenzel, M.; Hirsch, T.; Gutzmer, J.: Gallium, germanium, indium, and other trace and minor elements in sphalerite as a function of deposit type-A meta-analysis, Ore Geology Reviews, 77 (2016), S. 52-78

11. George, L.L.; Cook, N.J.; Ciobanu, C.L.: Partitioning of trace elements in co-crystallized sphalerite-galena-chalcopyrite hydrothermal ores, Ore Geology Reviews, 77 (2016), S. 97-116 
12. Schmid, S.M., et al.: Tectonic map and overall architecture of the Alpine orogen, Eclogae Geologicae Helvetiae, 97 (2004), S. 93-117

13. Schmid, S.M., et al.: Tectonic units of the Alpine collision zone between Eastern Alps and western Turkey, Gondwana Research, 78 (2020), S. 308-374

14. Schuster, R.: The Austroalpine crystalline units in the Eastern Alps, Berichte des Institutes für Erdwissenschaften der Karl-FranzensUniversität Graz, 9 (2004) ,S. 30-36

15. Melcher, F, et al.: The Haidbach deposit in the Central Tauern Window, Eastern Alps, Austria: a metamorphosed orthomagmatic NiCo-Cu-PGE mineralization in the Polymetallic Ore District Venediger Nappe System - Hollersbach Complex, Austrian Journal of Earth Sciences, 114 (2020), S. 1-26

16. Vavtar, F: Die Erzanreicherungen im Nordtiroler Stubai-, Ötztal-und Silvrettakristallin, in Arch. f. Lagerst.forsch. Geol. B.-A., 9 (1988), S. 103-153

17. Sangster, D.F.: Evidence for lateral fluid flow in vent-distal SEDEX $\mathrm{Zn}-\mathrm{Pb}$ deposits and districts, Mineralium Deposita, 55 (2019), S. 399-407

18. Sangster, D.F.: The role of dense brines in the formation of ventdistal sedimentary-exhalative (SEDEX) lead-zinc deposits: field and laboratory evidence, Mineralium Deposita, 37 (2002), S. 149-157

19. Southgate, P.N., et al.: A Basin System and Fluid-Flow Analysis of the $\mathrm{Zn}-\mathrm{Pb}-\mathrm{Ag}$ Mount Isa-Type Deposits of Northern Australia: Identifying Metal Source, Basinal Brine Reservoirs, Times of Fluid Expulsion, and Organic Matter Reactions, Economic Geology, 101 (2006), S. $1103-1115$

20. Leach, D.L., et al.: Sediment-hosted Pb-Zn Deposits: a global perspective, Economic Geology, 100 (2005), S. 561-608

21. Goodfellow, W.; Lydon, J.: Sedimentary exhalative (SEDEX) deposits, in Mineral deposits of Canada: A synthesis of major deposit types, district metallogeny, the evolution of geological provinces, exploration methods. 2007, Geological Association of Canada, Mineral Deposits Division, Special Publication, 5 (2007), S. 163-183

22. Niggli, M.: Geochemische Untersuchungen metamorpher Pb-Zn-Lagerstätten und ihrer Nebengesteine, Doktorarbeit, ETH Zürich, 1987

23. Artioli, G., et al.: Chemical and isotopic tracers in Alpine copper deposits: geochemical links between mines and metal, Geo. Alp 5 (2008), S. $139-148$
24. Hoinkes, G., et al.: Alpine metamorphism of the Eastern Alps, Schweizerische Mineralogische und Petrographische Mitteilungen, 79 (1999), S. 155-181

25. Hoinkes, G.; Purtscheller, F; Tessadri, R.J.G.P.M.: Polymetamorphose im Ostalpin westlich der Tauern (Ötztaler Masse, Schneeberger Zug, Brennermesozoikum), J Geol. Paläont. Mitt., 12 (1982), S. $95-113$

26. Konzett, J., et al.: Metamorphic evolution of iron-rich mafic cumulates from the Otztal-Stubai Crystalline Complex, Eastern Alps, Austria, Journal of Petrology, 46 (2005), S. 717-748

27. Koziol, M.; Oberhänsli, R.: Die südliche Eklogitzone im Ötztal, Mitteilungen der Österreichischen Geologischen Gesellschaft, 140 (1995), S. $95-117$

28. Miller, C.; Thöni, M.: Origin of eclogites from the Austroalpine Ötztal basement (Tirol, Austria): geochemistry and Sm-Nd vs. Rb-Sr isotope systematics, Chemical Geology, 122 (1995), S. 199-225

29. Thöni, M.: Degree and evolution of the Alpine metamorphism in the Austroalpine unit $\mathrm{W}$ of the Hohe Tauern in the light of $\mathrm{K} / \mathrm{Ar}$ and $\mathrm{Rb} / \mathrm{Sr}$ age determinations on micas, Jahrbuch der geologischen Bundesanstalt, 124 (1981), S. 111-174

30. Thöny, W.F., et al.: The metamorphic evolution of migmatites from the Ötztal Complex (Tyrol, Austria) and constraints on the timing of the pre-Variscan high-T event in the Eastern Alps, Swiss Journal of Geosciences, 101 (2008), S. 111-126

31. Mizuta, T.; Scott, S. D.: Kinetics of iron depletion near pyrrhotite and chalcopyrite inclusions in sphalerite; the sphalerite speedometer, Economic Geology, 92 (1997), S. 772-783

32. Brandner, R.: Tirol-Atlas, Geologische Ubersichtskarte von Tirol, Institut für Landeskunde, Universität Innsbruck, 1980

33. Haditsch, J.G.: Bemerkungen zur metallogenetischen Karte Tirols, Geol. Paläont. Mitt. Innsbruck, 20 (1995), S. 447-465

34. Keith, M., et al.: Effects of temperature, sulfur, and oxygen fugacity on the composition of sphalerite from submarine hydrothermal vents, Geology, 42 (2014), S. 699-702

35. Vavtar, F: Das Zinn-Kupfer-Erzvorkommen am Glücksgrat (StubaiKristallin), Tschermaks mineralogische und petrographische Mitteilungen, 35 (1986), S 287-298

Hinweis des Verlags. Der Verlag bleibt in Hinblick auf geografische Zuordnungen und Gebietsbezeichnungen in veröffentlichten Karten und Institutsadressen neutral. 